\title{
Equalization and Space Diversity Techniques in Mobile Environments
}

\author{
Under certain conditions, diversity techniques perform better than \\ equalization techniques in typical mobile radio environments.
}

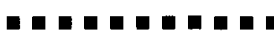

Antonio Valdovinos and Fernando J. Casadevall

FERNANDOJ. CASADEVALL is a full professor in the Signal Theory and Communications Department, Universitat Politecnica de Catalunya (UPC).

ANTONIO VALDOVINOS is an assistant professor at $U P C$.

\footnotetext{
${ }^{1} \mathrm{e}$ is the root meansquare delay spread (see expression 8) and $R_{b}$ is the bit rate.
}

I $\mathrm{n}$ the last few years, the development of high-quality digital mobile radio systems has become a major goal in the field of digital communications. The possibility of providing speech and data services free of wire constrictions in different environments, such as mobile vehicles, or within large buildings (hospitals, factories, department stores, etc.) is a very attractive proposition, supported by means of the personal communications network (PCN) concept. The aim of this article is to analyze the behavior of different receiver structures for such systems in order to obtain some clues to be used in designing the next generation of mobile systems. These systems have to provide both low- and high-data-rate services (up to $1 \mathrm{Mb} / \mathrm{s}$ ) and be efficient in environments where a high density of traffic is generated. Moreover, as the carrier frequencies will range between 1 and $4 \mathrm{GHz}$, and the vehicle speed can be as high as $400 \mathrm{~km} / \mathrm{hr}$ (e.g., high-speed trains), large Doppler frequency values must be coped with. On the other hand, PCN systems are power-limited due to portability constraints; therefore, coherent detection is preferable over differential detection, since a gain value of around $3 \mathrm{~dB}$ in terms of signal-to-noise ratio is achieved. For these reasons, $M$-quadrature amplitude modulation (QAM) schemes, which provide high spectrum efficiency and allow coherent detection, are appropriate for these systems. In particular, 4and 16-QAM schemes have been analyzed.

In these systems, the received signal is strongly affected by the radio environment. Buildings, hills, and other obstacles may obstruct the line of sight between transmitter and receiver. As a consequence of typical wave propagation characteristics such as scattering, diffusion, and diffraction, the resulting wave front in the receiving antenna consists of a number of time-varying multipath components. For this reason, mobile radio systems are affected by frequency-selective fading caused by the multipath time delay spread that produces both intersymbol interference (ISI) and Rayleigh fluctuations in the signal-to-noise ratio. The main effects of these phenomena on the system behavior are an irreductible bit error rate (BER) and the imposition of an upper limit on the data rate for which the system is able to guarantee a certain performance (e.g., the BER). In order to combat the degrading effects introduced by the channel, different protection techniques must be used. In particular, diversity techniques and/or adaptive channel equalizers could be considered.

All the techniques mentioned above are only able to fight properly against the propagation effects for a limited range of bit-rate values. Diversity techniques are efficient for $\operatorname{low}^{1}\left(\tau . R_{b} \ll 0.01\right)$ or medium $\left(0.01<\tau . R_{b}<0.1\right)$ bit rate transmission systems. In these cases, the transfer function of the channel can be assumed to be nonselective, and the ISI effects are negligible. Therefore, diversity techniques are able to combat the fluctuations in the signal-to-noise ratio due to the Rayleigh nature of the propagation. However, when the system operates at a high bit rate $\left(\tau . R_{b}>0.1\right)$, diversity cannot cope with the ISI; in this case, equalization techniques are required. Nevertheless, these techniques, which are capable of compensating the ISI, have limited performance due to the signal-to-noise fluctuations. Thus, to increase the system performance, it would also seem appropriate to consider joint diversity and equalization techniques, as analyzed in this article.

It must also be kept in mind that the objective of this article is only to analyze the improvement in system performance due to diversity and equalization techniques; therefore, all the results are given for uncoded systems. In a practical system, where some kind of coding is introduced, the BER can be reduced by more than four orders of magnitude, and this way, a suitable performance can be achieved.

Two different system configurations have been analyzed. First, we have considered a noise-limited system, where there is no cochannel interference. Second, a cellular radio environment has also been analyzed. In this case, the system performance becomes limited due mainly to cochannel interference rather than noise, since there are several base stations operating at the same frequency. In particular, six interfering signals corresponding to the first ring of cochannel interfering cells surronding the desired cell have been considered. 


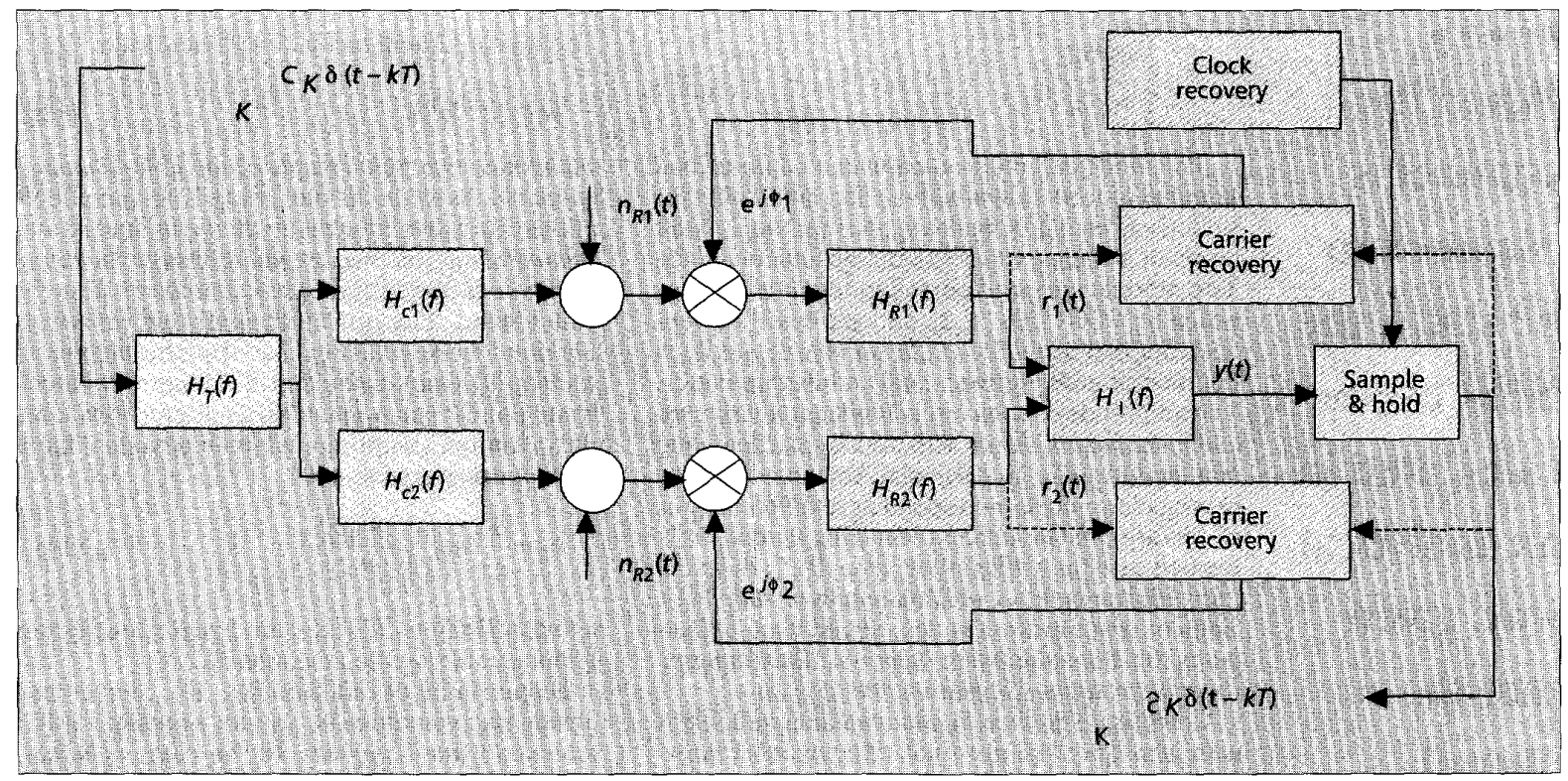

Figure 1. Low-pass equivalent model of the transmission system.

The influence on the system performance of various system and channel parameters, such as the shape and root mean square (RMS) delay spread of the power delay profile that characterizes the propagation conditions for different outdoor environments (urban, rural, and hilly terrains), the equalizer structure (linear transversal equalizer, LTE, or decision feedback equalizer, DFE), the number of taps, the introduction of a matched filter in the receiver, and so on, have been studied.

\section{System and Channel Models}

$\boldsymbol{F}$ igure 1 depicts the low-pass equivalent chanFel model of the transmission system under study.

In the following expressions, low-pass equivalent notation is used. In the most general case, the M-QAM generated signal can be expressed as

$$
\begin{aligned}
S(t) & =\sum_{k=-\infty}^{\infty}\left(a_{k}+j b_{k}\right) \cdot \delta(t-k T) \\
& =\sum_{k=-\infty}^{\infty} C_{k} \cdot \delta(t-k T)
\end{aligned}
$$

where $\left\{a_{k}\right\},\left\{b_{k}\right\}$ are independent data sequences of duration $T$ for the in-phase and quadrature channels, respectively. They are selected from the set $\left\{ \pm 1, \pm 3, \ldots, \pm\left(M^{1 / 2}-1\right)\right\}$ with $M=4$ for 4 -QAM and $M=16$ for 16 -QAM. $H_{T}(f)$ is the root-raised cosine filter and models the transmitted wave form. We have considered two different structures for the receiver filter, $H_{R}(f)$. In the first, the overall filtering transfer function, $H_{T}(f) \cdot H_{R}(f)$, is a raised-cosine type with a roll-off factor equal to 0.5 , and the filtering is split equally between the transmitter and receiver, which means that $H_{R}(f)$ is also the root-raised cosine filter. This filter is simpler than the matched filter and would be optimal if the channel transfer function were nonselective. In the second one, a matched filtering (MF) is incorporated, so

$$
h_{R}(t)=F^{-1}\left[H_{R}(f)\right]=h_{1}^{+}(-t)
$$

where + denotes the conjugate, $F^{-1}$ is the inverse Fourier transform, and $h_{1}(t)$ is the equivalent impulse response prior to $h_{R}(t)$,

$$
h_{1}(t)=h_{T}(t) * h_{c}(t)
$$

being

$$
h_{T}(t)=F^{-1}\left[H_{T}(t)\right]
$$

Therefore, the global impulse response results are

$$
\begin{aligned}
h(t) & =h_{T}(t) * h_{c}(t) * h_{R}(t)=h_{1}(t) * h_{1}^{+}(-t) \\
& =\alpha(t)=\alpha^{+}(-t)
\end{aligned}
$$

Let us notice that the receiver filter is matched not only to the root-raised cosine transmitting filter, but also to the entire impulse response $h_{1}(t)$, which is affected by the channel impulse response variations, and thus needs to be continually updated. It could be implemented according to an FIR structure from the estimated impulse response. The function $h_{c}(t)$ models the channel behavior that introduces selective fading in the radio link. The channel is assumed to be wide-sense stationary uncorrelated scattering (WSSUS), and it is represented by a unique correlation function referred to as the Power Delay Profile $P(t)$, given by:

$$
<h c(t) h^{*}\left(t^{\prime}\right)>=P(t) \delta\left(t-t^{\prime}\right)
$$

where $<$. $>$ denotes the ensemble average, and

$$
\mathrm{P}(\mathrm{t})=\left\langle\left|h_{c}(t)\right|^{2}\right\rangle
$$

A measure of the width of $P(t)$ is the RMS delay spread, $\tau$, defined as

$$
\tau=\left[\frac{\int\left(t-\tau_{m}\right)^{2} P(t) d t}{\int P(t) d t}\right]^{\frac{1}{2}}
$$

where the average delay $\tau_{m}$ is defined as 
a $\mathbf{\square}$

When the

channel is

assumed to

be static, the

BER can be

calculated in

an analytical

way, given

that the

overall

impulse

response is

estimated,

and thus,

the ISI

component

known.

$$
\tau_{m} \frac{\int t P(t) d t}{\int P(t) d t}
$$

The multipath phenomenon can be described in terms of time delays with no Doppler shifts for static channels, and time delays and the Doppler shift associated with each delay for the dynamic channels. The models adopted correspond to those proposed by COST-207 $[1,2]$, and are based on representing the channel by a finite number of taps, each one determined by its time delay and average power. The amplitude of each tap is Rayleigh distributed and varies in accordance with the Doppler spectrum (see Appendix I). In particular, the four analyzed propagation modelscorresponding todifferent types of terrain are the urban (non-hilly) area (TU), the bad case for a hilly urban area (BU), the rural (non-hilly) area (RA), and finally, hilly terrain (HT). Each of these models is described by a continuous power delay profile (see Appendix II) and approximated through a discrete number of taps.

Considering the most general case analyzed, which is to introduce the presence of cochannel interference, the received signal for each diversity branch may be expressed as

$$
\begin{aligned}
r(t) & =\sum_{k=-\infty}^{\infty}\left[a_{k} \cdot h^{R}(t-k T)-b_{k} \cdot h^{I}(t-k T)\right] \\
& +j \sum_{k=-\infty}^{\infty}\left[b_{k} \cdot h^{R}(t-k T)+a_{k} \cdot h^{I}(t-k T)\right] \\
& +i(t)+n_{x}(t)+j n_{y}(t)
\end{aligned}
$$

where $i(t)$ is the cochannel interfering signal (ICI) and

$$
\begin{aligned}
h(t) & =h^{R}+j h^{I} \\
& \left.=F^{-1}\left[H_{T}(f) \cdot H_{R}(f)\right]^{*} h_{c}(t)\right] \cdot G \cdot e^{-j \theta}
\end{aligned}
$$

with * the convolution operator. $G$ is a gain factor that ensures a constant average power at the demodulator input. It is introduced in order to consider the presence of automatic gain control (AGC) and is only necessary for 16-QAM patterns since in 4-QAM we have zero-crossing decision boundaries. After some algebraic effort, it is easy to show that $i(t)$ may be expressed as follows:

$$
\begin{aligned}
i(t) & =\sum_{m=1}^{6} \alpha_{m} \sum_{k=-\infty}^{\infty}\left[a_{k, m} h_{m}^{R}\left(t-\xi_{m}-k T\right)\right. \\
& \left.-b_{k, m} h_{m}^{I}\left(t-\xi_{m}-k T\right)\right] \\
& +j \sum_{m=1}^{6} \alpha_{m} \sum_{k=-\infty}^{\infty}\left[b_{k, m} h_{m}^{R}\left(t-\xi_{m}-k T\right)\right. \\
& \left.+a_{k, m} h_{m}^{I}\left(t-\xi_{m}-k T\right)\right]
\end{aligned}
$$

where $\alpha_{m}$ is equal to the inverse of the carrier-tointerference ratio (CIR) in linear unities, $\xi_{m}$ is a random variable that represents the difference in timing phases between the interfering and desired signals, which has a uniform distribution over $[0, T]$, and $h_{m}(t)$ is the corresponding equivalent impulse response for each of the six interfering signals.

In order to perform a coherent detection, we have introduced a carrier recovery circuit that minimizes the output mean square error. Assuming that the bandwidth of this circuit is much higher than the fading rate, the carrier phase can be properly tracked.
Then the optimum recovered phase results [3]:

$$
\varphi_{\text {opt }}=-\arg \left(h_{0}\right)
$$

where $h_{0}$ is the main sample of the global impulse response.As we focus our analysis on the effects of the distortion induced by the delay spread and cochannel interference, the phase jitter on the recovered carrier caused by the Gaussian noise will not be considered. The optimum sampling instant is obtained from a classical squaring timing recovery loop [4]. Considering that the fading rate is much lower than the bandwidth of the timing recovery loop, the optimum sampling instant is obtained from the following expression:

$$
t_{d}=\frac{T}{2 \pi} \tan ^{-1}\left[\frac{H^{I}(f)}{H^{R}(f)}\right]_{f=1 / T}
$$

where $\left[H^{R}(f)+j H^{I}(f)\right]$ is the Fourier transform of the global impulse response $h(t)$.

It should be noted here that the carrier and timing recovery circuits are not necessary if the matched filter is present. In this case, $h_{0}$ is real, and therefore, the optimum recovered phase is always zero. In addition, since the maximum of $h(t)$ is in the middle point of this function, $t_{d}=0$.

Two baseband equalizer structures are used. The system performance for typical LTE and DFE structures, both with symbol period $(T)$ spacing between stages, have been investigated. In both cases, the minimum mean square error (MMSE) technique has been adopted to calculate the tap values. When static radio channels are assumed, a set of linear equations can be posed to obtain the optimum tap values. The equations to be solved are different depending on the situation we are dealing with: ICI effect [5], equalization only, or joint equalization and diversity [6]. For dynamic channels, we first estimate the channel impulse response from the midamble sequence, which is known in the receiver; afterwards, a recursive least squares (RLS) updating procedure is adopted to follow the time-variant channel characteristics.

\section{Results}

WT have examined the effectiveness of the joint adaptive equalization and diversity techniques in counteracting the multipath and fading introduced by the mobile radio channel as well as the cochannel interference due to the cellular environment. The objective is to determine the data rate limitation for mobile communication systems. In order to analyze a standard time-division multiple access (TDMA) system, we have obtained results considering a burst structure like that adopted by the GSM system [7]. The criterion used to evaluate the system quality is the BER. When the channel is assumed to be static, the BER can be calculated in an analytical way, given that the overall impulse response is estimated, and thus, the ISI component known. To compute the error probability integral, we have considered the Levy method [8]. With regard to the dynamic channel, the impulse response is also estimated, but it changes each bit period, and the use of an analytical method would involve an inadmissible simulation time. For this reason, the traditional Montecarlo method has been used. In particular, the average 


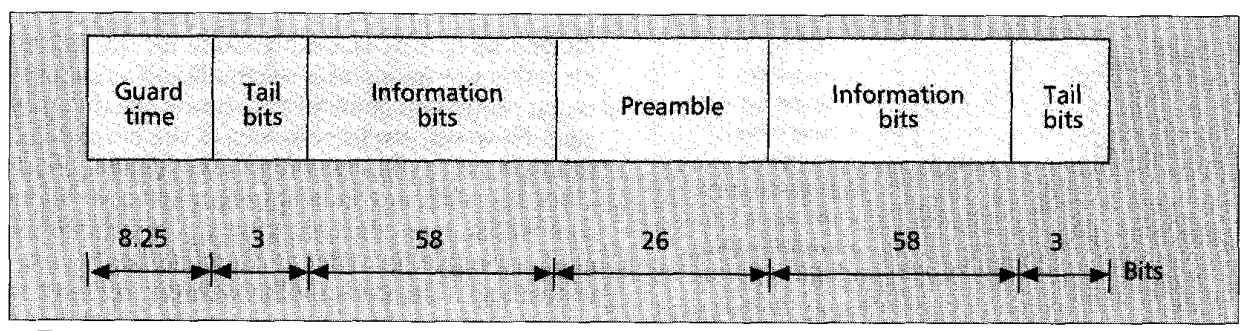

Figure 2. Basic time slot structure.

BER has been obtained from 10,000 bursts, each consisting of $148 \mathrm{~b}$, as shown in Fig. 2.

Furthermore, in order to compare the performance of the two different modulation schemes analyzed, we have used the bit rate $R_{b}$ as the normalization parameter. The BER value is expressed as a function of the normalized delay spread given by the product $\tau R_{b}$; that is, if we want to know what the maximum bit rate is for a given value of BER, we just have to know what the RMS delay spread value is to compute the maximum $R_{b}$ value.

Throughout this presentation of results, we will focus mainly on the TU model, because it represents a typical environment in which a $\mathrm{PCN}$ system could be deployed and a high density of traffic expected.

In Figs. 3a and 3bwe show the evolution of the mean value of the bit-error probability for 4- and 16-QAM, respectively. We have chosen signal-to-noise ratio values of 20 and $26.5 \mathrm{~dB}$ for 4 - and 16-QAM, respectively, because they provide a similar BER value for systems without ISI and affected by Rayleigh fading. In this case, a static TU channel model has been considered and a matched filter introduced. From the comparison of these figures, it can be seen that the BER evolution is similar, so the same conclusions can be obtained from both figures. It is clear that an unprotected system performs very poorly, even for channels with low distortion. Certainly, the Rayleigh fading induced by the multipath propagation significantly degrades the system performance, even if there is no ISI. For the assumed signal-to-noise ratio value and 4-QAM pattern, the BER has a value of $5.10^{-3}$ in Rayleigh environments, while in a Gaussian channel the BER is as low as $7.10^{-24}$. In fact, the system is only able to guarantee a BER value lower than $10^{-2}$ if the normalized delay spread does not exceed 0.2 and 0.15 for 4 -and 16 -QAM patterns, respectively. If the diversity technique is considered, we notice that the BER values decrease by up to one order of magnitude in the event that the channel introduces low distortion, as could be expected in Rayleigh environments [9]. However, for highervalues of $\tau . R_{b}$, the system performance degrades quickly and proves to be slightly better than that obtained for an unprotected system. In thiscase, we can achieve BER values lower than $10^{-3}$ for $\tau . R_{b}$ values lower than 0.25 and 0.18 for $4-$ and 16-QAM, respectively. If LTE and DFE structures are introduced, the system performance increases quickly due to the ability of the equalizer to compensate the distortion induced by the channel. For an equalizer with three taps (marked $2+1$ in the figure), the system is able to guarantee a BER lower than $10^{-2}$ for values of the normalized delay spread as high as 1 for LTE and 1.4 for DFE when 4-QAM is considered ( 0.4 and 0.7 , respectively, for 16-QAM). Moreover, if the number of taps is increased to seven (marked $4+3$ in the

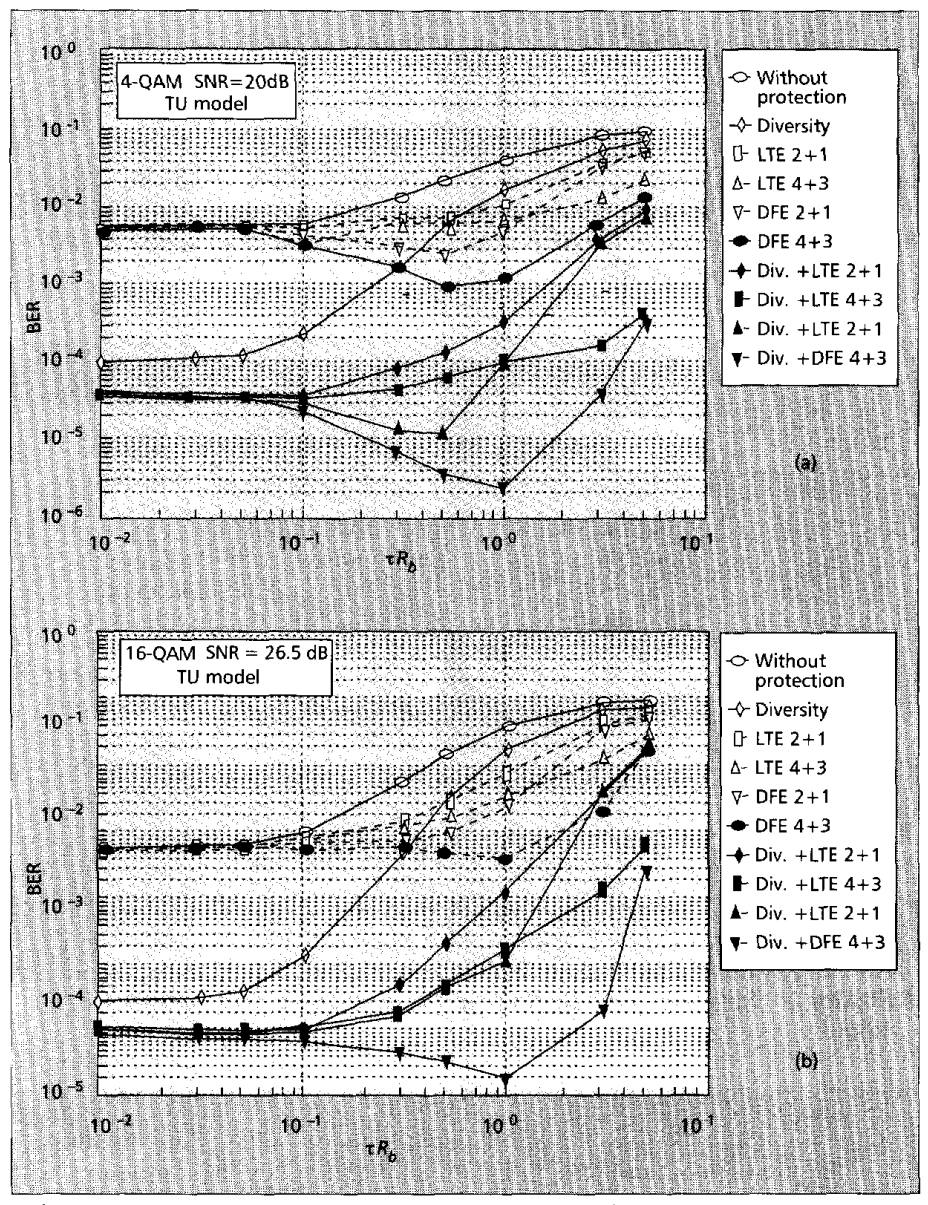

Figure 3. Evolution of the BER versus the normalized delay spread for the TU model: (a) 4-QAM; (b) 16-QAM.

figure), the BER remains lower than the above value if $\tau . R_{b}$ is kept lower than 2 and $4(0.5$ and 3 for 16-QAM) for LTE and DFE structures, respectively. On the other hand, when joint equalization and diversity techniques are considered, we obtain a marked improvement in performance because we are able to compensate for both the induced distortion and the degradation in terms of signal-tonoise ratio due to the Rayleigh fading. In this case, for 4-QAM a BER value of $10^{-4}$ can be achieved for normalized delay spreads lower than 0.35 for LTE $(2+1), 1$ for $\operatorname{LTE}(4+3), 1$ for DFE $(2+1)$, and 4 for DFE $(4+3)$ (for 16-QAM, the respective values are $0.2,0.4,0.4$, and 3 ). It is important to emphasize that for some $\tau . R_{b}$ values, the system performance is better than that obtained for a channel that 


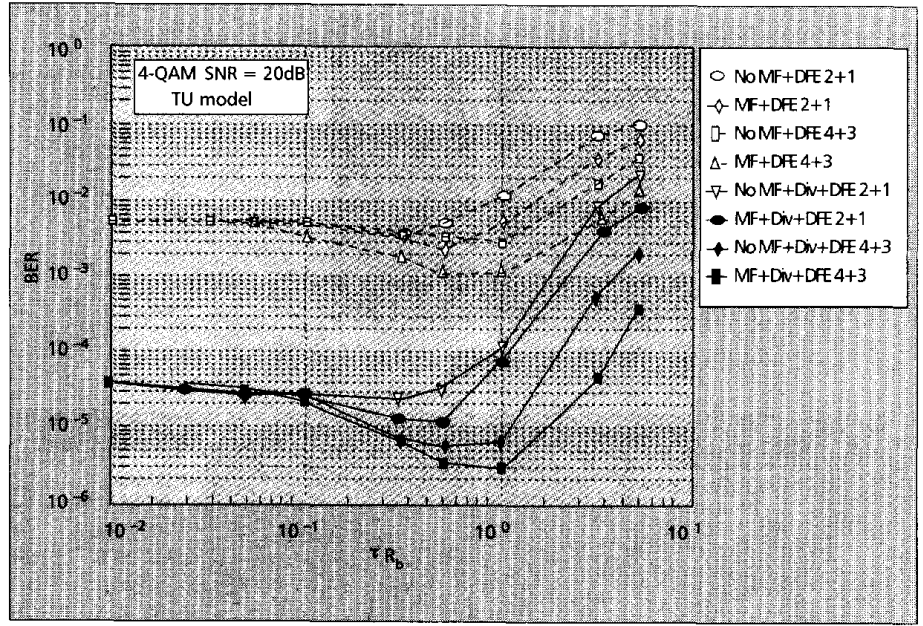

Figure 4. Evolution of the BER versus the normalized delay spread for $M F$ and root raised cosine filter.

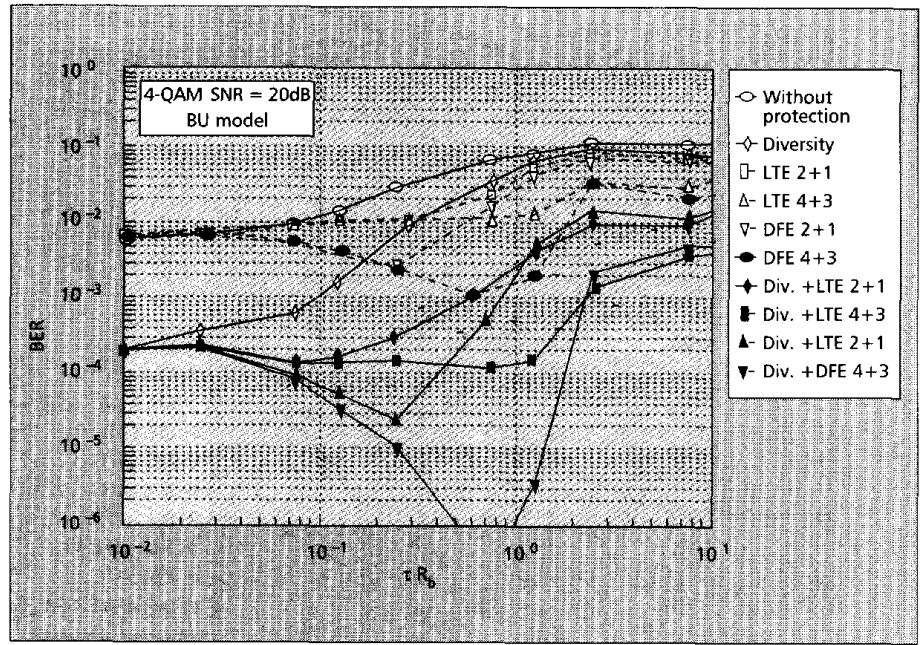

Figure 5. Evolution of the BER versus the normalized delay spread, $B U$ model.

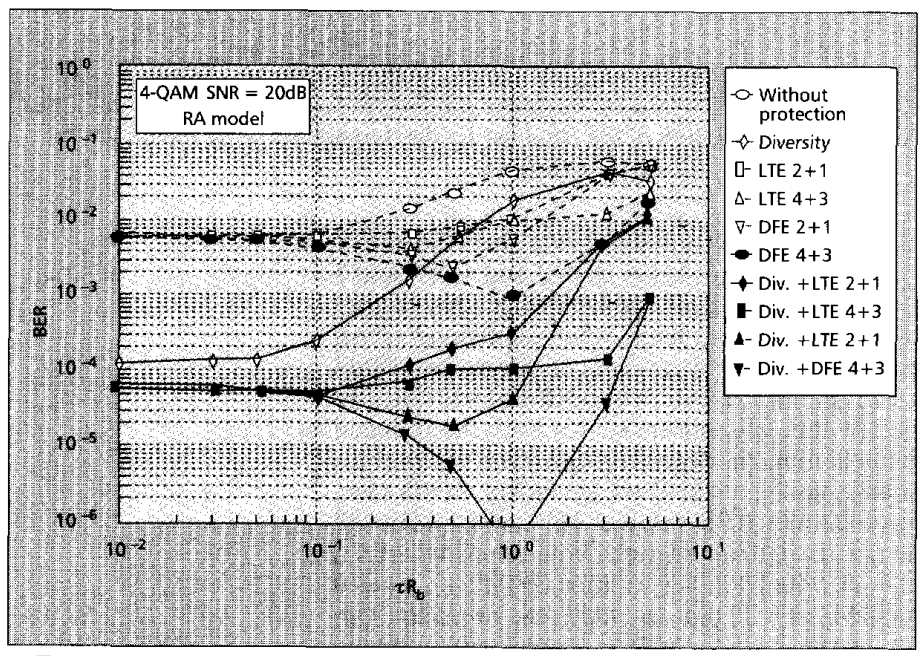

Figure 6. Evolution of the BER versus the normalized delay spread, $R A$ model. introduces no ISI. The explanation for this behavior is that the system uses the multipath as an additional redundant channel to increase the diversity gain. However, for large values of the normalized delay spread, the ISI induced by the multipath increases considerably, and the equalizer cannot completely cope with it. As a result, the system performance degrades quickly. In order to illustrate the bit-rate improvement introduced by the use of diversity and/or equalization techniques, a typical value of $\tau=1$ $\mu \mathrm{s}$ is assumed for the TU model. From Fig. 3a, to achieve a BER equal to $10^{-3}$, a system with diversity alone is able to transmit up to $200 \mathrm{~kb} / \mathrm{s}$, approximately $\left(\tau \cdot R_{b}=0.2\right)$. If only equalization techniques are used, the system is not capable of guaranteeing this value of $B E R$, but considering joint equalization and diversity, we can reach up to $6 \mathrm{Mb} / \mathrm{s}$.

Finally, as a comparison between 4- and 16-QAM schemes, the results obtained show that the use of high-level $M$-QAM modulations offers no advantage in terms of the maximum data rate that can be transmitted. Certainly, even though narrow-spectrum bandwidth results when high-order modulations are considered, and, as a consequence, a minor effect of the multipath propagation could be expected, the great sensitivity to the distortion of the highorder modulation causes no advantage to be achieved. Consequently, high-level $M$-QAM schemes could only be considered in order to obtain high spectrum efficiency, but not because of their capability to cope with large time dispersion values introduced by multipath propagation.

We have also analyzed the benefits of using a matched filter in place of a root-raised cosine filter in the receiver. For all the various cases analyzed, Fig. 4 shows that when the matched filter is considered, the BER curve obtained is clearly below that corresponding to the root-raised cosine filter. Therefore, we will always consider the presence of a matched filter from now on.

The BER evolution for the different static channel models is illustrated in Figs. 5, 6, and 7 for $\mathrm{BU}, \mathrm{RA}$, and $\mathrm{HT}$, respectively. Both the analysis of the results obtained and the conclusions are similar to those explained above for TU environments. In order to compare the maximum data rate allowed in those environments, it must be said that the typical $\tau$ values for each of these models are $2.5,0.1$, and $5 \mu \mathrm{s}$, respectively. Therefore, in order to guarantee a BER lower than $10^{-3}$, maximum bit rates of $0.8,40$, and $0.5 \mathrm{Mb} / \mathrm{s}$ can be achieved, respectively, assuming joint diversity and $4+3$ DFE equalization.

Figure 8 shows the system performance for a dynamic channel as a function of the signal-to-noise ratio. In particular, a Doppler frequency of $250 \mathrm{~Hz}$ and a bit rate of $500 \mathrm{~kb} / \mathrm{s}$ have been chosen. We observe a great improvement in terms of signal-to-noise ratio due to the use of joint diversity and equalization. For a BER value equal to $10^{-3}$, using a DFE equalizer with seven taps and diversity, we gain up to $13 \mathrm{~dB}$ in relation to the equalizer without diversity. We also notice that the DFE structure presents a gain of about $5 \mathrm{~dB}$ in relation to the LTE.

If we compare these results for a SNR equal to $20 \mathrm{~dB}$ with those obtained in Fig. $3 \mathrm{a}$ for $\tau . R_{b}$ equal to $0.5\left(\tau=1 \mu \mathrm{s}\right.$ and $\left.R_{b}=500 \mathrm{~kb} / \mathrm{s}\right)$, only small differences appear. Therefore, we can conclude that if the system is able to obtain the channel impulse response from the midamble data, an 
RLS tap-updating algorithm can be used to cope with the time-variant fluctuations of the channel impulse response without a significant system performance penalty. Thus, for the TDMA burst structure analyzed, all the results obtained for static channels can be extended to the dynamic case. Let us notice that the use of a midamble instead of a preamble simplifies the updating algorithm complexity because the effect of channel fluctuation with which the receiver has to cope are minimized, Certainly, with a midamble the last symbols of the burst are closer to the point where the impulse response is estimated.

Finally, Fig. 9 shows the system behavior when the performance is limited by the cochannel interference. We have considered a signal-tonoise value of $60 \mathrm{~dB}$ and a global carrier-to-interference ratio $(\mathrm{C} / \mathrm{I})$ of $20 \mathrm{~dB}$. It should be noted that now the system performance is mainly limited by the interference rather than by the noise. Six independent interfering signals have been generated. In this case, for a BER of $10^{-3}$, and assuming joint equalization and diversity, we can reach normalized delay spreads higher than 5 . That is, for a TU environment a maximum bit rate as high as 7 $\mathrm{Mb} / \mathrm{s}$ could be expected, keeping the BER lower than $10^{-3}$. We also notice that the system has a behavior very similar to that obtained for a noise-limited system, albeit with slightly better performance. In fact, if we had established a Gaussian hypothesis for the cochannel interference, which represents the worst case, we would have obtained the same results as for the noise-limited system with a signal-to-noise ratio of $20 \mathrm{~dB}$. Thus, the results obtained for noise-limited systems with the environments considered could be extended to systems limited by cochannel interference.

It should be emphasized that although the BER values obtained in this analysis may seem too high, as we have stated before, we have only focused on the improvement obtained by using equalization and/or diversity techniques. However, a practical system also introduces coding and interleaving; as a result, the final BER could be found to be more than four orders of magnitude below the values presented in this article.

\section{Conclusions}

$\mathrm{T}$ this article we have analyzed the performance of 1 diversity and equalization techniques in a mobile radio environment for both noise- and cochannel-interference-limited systems. Four- and 16-QAM modulations have been considered, and several realistic outdoor channel models have been described. From the results obtained, we can conclude that a system without protection has very limited performance. When the channel introduces low-level distortion, the diversity technique produces good performance; on the other hand, when the channel introduces a high degree of ISI, the use of equalization techniques becomes necessary. In particular, the DFE structures offer better performance than the LTE ones. Moreover, as the tap values of the feedback filter can be obtained, using a simple expression [10], from the known impulse response and the linear coefficients, it can be concluded that with the same number of taps, the DFE also improves on LTE performance from the point of view of computing complexity. This

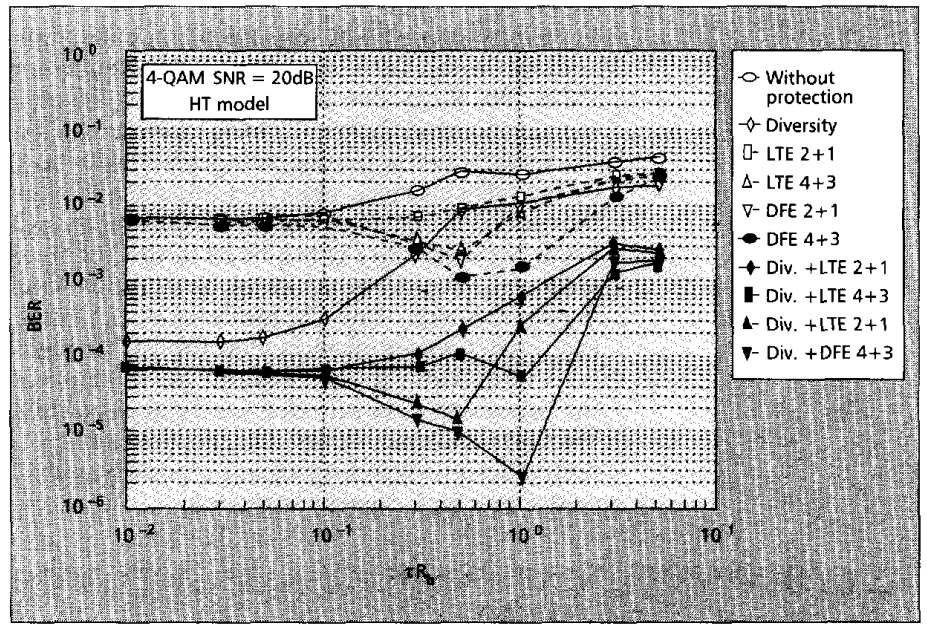

Figure 7. Evolution of the BER versus the normalized delay spread, $H T$ model.

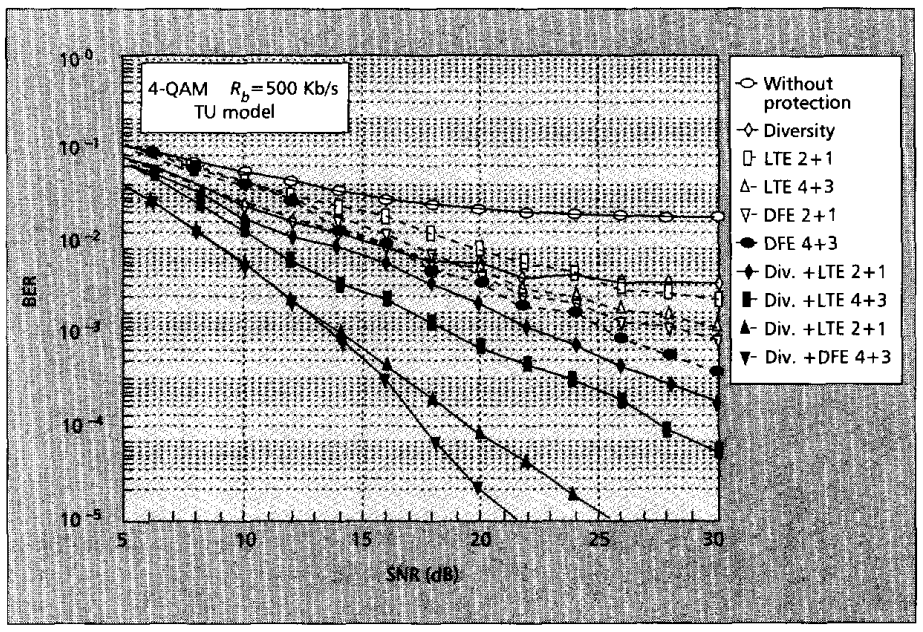

Figure 8. Evolution of the BER versus the nignal-to-noise ratio for a dynamic channel. Doppler frequency $\mathrm{f}_{\mathrm{d}}=250 \mathrm{~Hz}$.

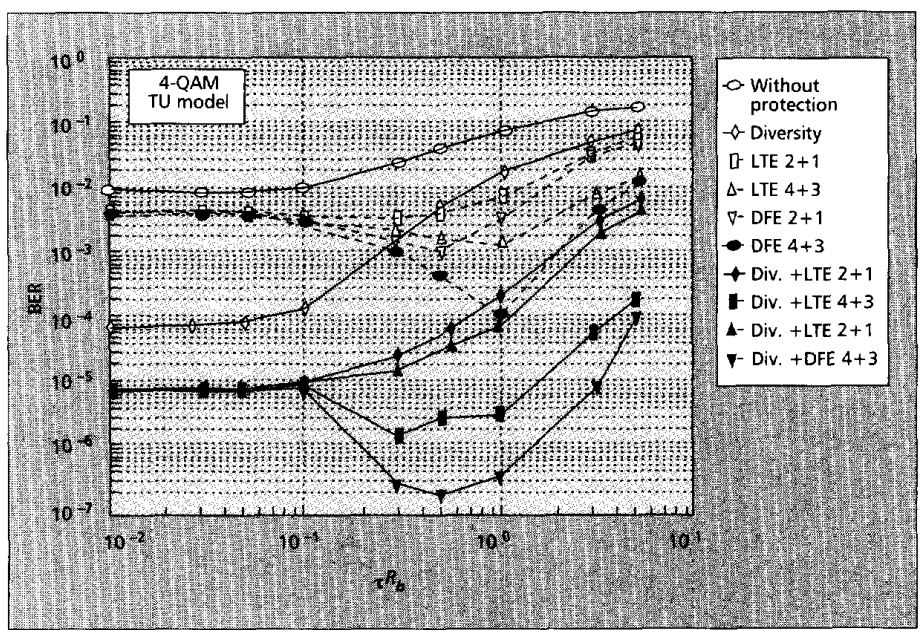

Figure 9. Evolution of the BER versus the time delay spread. Cochannelinterference-limited system. 
is an important point when dealing with highspeed transmission systems, as envisaged for PCN. We have also demonstrated that joint equalization and diversity techniques are very effective tools to combat the degrading effect introduced by the radio channel, since they allow the BER to be reduced by a factor of more than 100 , even though greater complexity is required in the receiver structure. On the other hand, as a comparison between 4- and 16-QAM modulation schemes, the results obtained show that the use of highlevel $M$-QAM offers no advantages in terms of the maximum data rate that can be transmitted. We must remember, though, that they are more spectrally efficient, which is significant, especially in small cells where high capacity is needed.

\section{Appendix I}

The system model is based on representing the channel by a discrete number of taps, each determined by its time delay and average power. The amplitude of each tap varies in aecordance with a Doppler spectrum. In particular, we have considered four types of Doppler spectra, which are defined in this section. The following abbreviations will be used:

- $f_{d}=v / \lambda$ represents the maximum Doppler shift, where $v(\mathrm{~m} / \mathrm{s})$ is the vehicle speed and $\lambda(m)$ the wavelength.

- $G\left(a, f, f_{2}\right)$ is the Gaussian function

$$
G(f)=A \exp \left[-\frac{\left(f-f_{1}\right)^{2}}{2 f_{2}^{2}}\right]
$$

The defined Doppler spectra are as follows. CLASS is the classical Doppler apectrum and is used for paths with delays not in excess of $500 \mathrm{~ns}$ $\left(\tau_{i} \leq 0.5 \mu s\right)$.

$$
\left.S(\tau, f)=\frac{A}{\sqrt{1-\left(\frac{f}{f_{d}}\right)^{2}}} \text { for } f \in\right]-f_{d,} f_{d}
$$

GAUS1 is the sum of two Gaussian functions, and it is used for paths with delays in the range of 500 as to $2 \mu \mathrm{s}\left(0.5 \mu \mathrm{s}<\tau_{i} \leq 2 \mu \mathrm{s}\right)$.

$$
\begin{aligned}
S(\tau, f) & =G\left(A,-0.8 f_{d}, 0.05 f_{d}\right) \\
& +G\left(A_{1}, 0.4 f_{d}, 0.1 f_{d}\right)
\end{aligned}
$$

where $A_{1}$ is $10 \mathrm{~dB}$ below $A$.

GAUS2 is also the sum of two Gaussian functions and is used for paths with delays in excess of $2 \mu s\left(\tau_{i} \geq 2 \mu s\right)$.

$$
\begin{aligned}
s(\tau, f)=G\left(B, 0.7 f_{d}, 0.1 f_{d}\right) \\
+G\left(B_{1},-0.4 f_{d i}, 0.15 f_{d}\right)
\end{aligned}
$$

where $B_{1}$ is $15 \mathrm{~dB}$ below $B$.

The values of $A$ and $B$ are obtained from

$$
P\left(\tau_{i}\right)=\int_{f_{f}} S^{2}\left(\tau_{i}, f\right) d f
$$

where $P\left(\tau_{i}\right)$ is the power transmitted by tap $i$ of a transversal filter.

RICEis the sum of a classical Dopplerspectrumand one directpath. Thisspectrum is used for the shortest path of the propagation model in rural areas.

$$
\begin{aligned}
S\left(\tau_{i}, f\right) & =\frac{0.17}{\pi f_{d} / 1-\left(\frac{f}{f_{d}}\right)^{2}} \\
& \left.+0.83 \delta\left(f-0.7 f_{d}\right) \text { for } f \in\right]-f_{d}, f_{d}
\end{aligned}
$$

\section{Acknowledgments}

This article reflects part of the work supported by CICYT (Spain) under Grant TIC 714/90.

\section{References}

[1] COST 207, "DigitalLand Mobile Radio Communications," Final Report Comm of the Europecan Communities, Luxembourg, pp. 135, 147, 1989 [2] R. W. Lorentz, "Corrections of COST 207 Proposals for Simulation of Radio Channels. " COST 231 TD (91) 26, Darmstadt, Feb. 1991 [3] S. Moridi and H. Sari, "Analysis of Decision-Feedback Carrie Recovery Loops with Applications to 16 QAM Digital Radio Systems, Int'1. Coni. Commun. (ICC '83), pp. 671-675, 1983.

[4] N. Amitay and L. J. Greenstein, "Multipath Outage Performance of Digital Radio Receivers Using Finite-Taps Adaptive Equalizers," IEE Trans. Commun., vol. COM-32, no. 5, pp. 479-481, May 1985

[5] F. J. Casadevall and A. Valdovinos, "Equalization Techniques in Indoor Radio Environments," EUSIPCO'92, Brussels, Belgium, pp. 139, 142. Aug. 24-27, 1992

[6] F. J. Casadevall and A. Valdovinos, "Performance of M-QAM Modulations for Indoor Radio Communications with Combined Diversity and Equalization Techniques," Int'l. J. Digital and Anaiog Commun. Sys., vol. 5, pp. 117-127, 1992.

[7] M. Moully and M. B. Pcutet, The GSM System for Mobile Communications, published by the cuthors, 1992

[8] A. Levy. "Fast Error Evaluation in the Presence of Intersymbol Interference," IEEE Trans. Commun., vol. COM-33, no. 5, pp. 479-81, 1985 [9]W.C.Y.Lee,MobileCommunications Engineering, (New York McGrowHill, 1982) [10]].G. Proakis, Digital Communications, (New York: McGraw Hill, 1983)

\section{Biographies}

FERNANDO]. CASADEVALL [M '87] received Engineer of Telecommunications and $\mathrm{Ph} . \mathrm{D}$. degrees from the Escola Tecnica Superior d'Enginyers de Telecomunicacio de Barcelona (ETSETB), Universitat Politecnica de Catalunya (UPC) in 1977 and 1983, respectively. In 1978, he joined the ETSETB, where he was an associate prolessor from 1983 to 1991. He is currenty aPC prossor in the Signal Theory and Communicatons Department. UPC. Barcelona, Spain. His research interests include digital communication with particular emphasis on equalization techniques applied to digital radio and its performance analysis under multipath propagatio conditions, cellular and personal communication systems, multipath receiver design, and digital signal processing. He is actively participating in the European research programs COST 231 and RACE.

ANTONO VALDOVINOS [S ' 91 ] received his Engineer of Telecommunication degree from the ETSETB, UPC, in 1990. In 1991, under a research grant he joined the Signal Theory and Communications Department, UPC. Barcelona, Spain, where he is currently an assistant professor and is pursuing his $\mathrm{Ph}$ D in the area of mobile rodio communicotions systems.

\section{Appendix II}

The propagation models are defined by the power delay profiles

$$
P\left(\tau_{i}\right)=\int_{-f_{d}}^{f} s^{2}\left(\tau_{i}, f\right) d f
$$

where $P(\tau)$ is the power transmitted by tap $i$ of a transwersal filter.

The continuous power delay profiles $P(\tau)$ are defined as follows ( $\tau$ in $\mu$ s):

- Rural (non-billy) area (RA).

$$
P(\tau)= \begin{cases}\cos ^{2}(-0.2+1) & \text { for } 0<\tau<0 ? \\ 0 & \text { elsew bere }\end{cases}
$$

- Urban (non-hilly) area (TU).

$$
P(\tau)=\left\{\begin{array}{cl}
\exp (\tau) & \text { for } 0<\tau<\tau \\
0 & \text { elsewhere }
\end{array}\right.
$$

- Bad case for hilly urban area (BU).

$$
P(\tau)= \begin{cases}\exp (-\tau) & \text { for } 0<\tau<5 \\ 0 S^{*} \exp (5+\tau) & \text { for } 15<\tau<10\end{cases}
$$

$P(\tau)=\left\{0 S^{*} \exp (5+\tau) \quad\right.$ for $15<\tau<10$

- Hilly terrain (HT)

$$
P(\tau)=\left\{\begin{array}{cc}
\exp \left(-35^{*} t\right) & \text { for } 0<\tau<2 \\
01 \exp (15-\tau) & \text { for } 15<\tau<20 \\
0 & \text { elsewhere }
\end{array}\right.
$$

The relationship between bothdiscrete and continuous power delay profiles is fully described in [1] 